\title{
Near-Optimal Parameterization of the Intersection of Quadrics
}

\author{
Laurent Dupont \\ LORIA - Université Nancy 2 \\ Campus Scientifique, B.P. 239 \\ 54506 Vandoeuvre-les-Nancy, France \\ Laurent.Dupont@loria.fr \\ Sylvain Lazard \\ LORIA - INRIA Lorraine \\ Campus Scientifique, B.P. 239 \\ 54506 Vandoeuvre-les-Nancy, France \\ Sylvain.Lazard@loria.fr
}

\author{
Daniel Lazard \\ LIP6 \\ Université Paris 6 \\ 75252 Paris, Cedex 05, France \\ Daniel.Lazard@lip6.fr \\ Sylvain Petitjean \\ LORIA - CNRS \\ Campus Scientifique, B.P. 239 \\ 54506 Vandoeuvre-les-Nancy, France \\ Sylvain.Petitjean@loria.fr
}

\begin{abstract}
In this paper, we present the first exact, robust and practical method for computing an explicit representation of the intersection of two arbitrary quadrics whose coefficients are rational. Combining results from the theory of quadratic forms, linear algebra and number theory, we show how to obtain parametric intersection curves that are near-optimal in the number and depth of radicals involved.
\end{abstract}

\section{Categories and Subject Descriptors}

F.2.2 [Analysis of Algorithms and Problem Complexity]: Nonnumerical Algorithms and Problems-Geometrical problems and computations; I.1.2 [Symbolic and Algebraic Manipulation]: Algorithms; I.3.5 [Computer Graphics]: Computational Geometry and Object Modeling -Boundary representations, Constructive solid geome$\operatorname{try}(C S G)$

\section{General Terms}

Algorithms.

\section{Keywords}

Robustness of geometric computations, quadric surface intersection.

\section{INTRODUCTION}

In solid modeling, the two most widely used types of object representation are constructive solid geometry (CSG) and boundary representation (BRep). Both representations having their own respective advantages, solid modeling kernels often need an efficient and reliable way to switch from the CSG to the BRep representation. CSG-to-BRep conversion, also known as boundary evaluation, is a well understood problem. However, past approaches have often put more emphasis on efficiency than on robustness and accuracy. Most current modelers use only finite-precision arithmetic for CSG-to-BRep conversion. The topological consistency of the computed BRep can easily be jeopardized by small amounts of error in the data introduced by finite-precision computations. For many applications in design and automated manufacturing, where topological consistency and accuracy are critical, this may be unacceptable.

Designing robust algorithms is currently a major interest of the computational geometry and geometric computing research communities (see, e.g., [3, 10, 13, 15, 24]). A number of approaches have been proposed for the robust and accurate boundary evaluation of polyhedral models $[2,9]$. Most rely heavily on numerical computation, with varying dependence on exact and floating-point arithmetic. Computing the topological structure of a BRep involves accurate evaluation of signs of arithmetic expressions. Assuming the input data has a bounded precision and allowing whatever bitlength is necessary for number representation, these signs can be computed exactly.

By contrast, there has been much less work on robust CSG-to-BRep conversion algorithms for curved primitives. A major reason is that, outside the linear realm, exact arithmetic computations require algebraic numbers, such as square roots, which cannot in general be represented explicitly with a finite number of bits. Also, computing with algebraic numbers is currently extremely slow.

One notable exception is the work of Keyser, Krishnan and Manocha [16] and Keyser, Culver, Foskey and Krishnan [14] on the boundary evaluation of low-degree CSG solids specified with rational parametric surfaces. The authors use exact arithmetic, present compact data structures for representing the boundary curves as algebraic curves and the boundary vertices as algebraic numbers and use efficient algorithms for computing the intersection curves of parametric surfaces. 
The quadratic nature of the equations defining quadric surfaces permits an explicit representation of their intersection curves. In other words, it is theoretically possible to compute a fully parametric representation of the boundary of quadric-based solids. The only known general method for computing a parametric representation of the intersection between two quadrics is well known and due to J. Levin [18, 19]. It is based on an analysis of the pencil of the quadrics.

Levin's seminal work has been extended in many different directions. Arguing that the pencil method does not yield explicit information on the morphological type of the intersection curve, Farouki, Neff and O'Connor [7] made a complete theoretical study of degenerate cases for general quadric surfaces. Goldman and Miller [11] took a different path and developed a special-case solution for each of the possible pairs of natural quadrics (i.e., planes, right cones, circular cylinders and spheres), later proving that the degenerate conic sections can be detected exactly by evaluating a few simple algebraic expressions [20]. Shene and Johnstone [23] have also worked on the lower degree intersections of natural quadrics.

Most of these methods were motivated by the fact that Levin's general method for intersecting implicit quadric surfaces is not numerically robust and may fail in degenerate configurations. Moreover, if exact arithmetic is used, either the calculations are impossible to complete or the method outputs parametric representations that are barely usable in applications, because of the high-degree algebraic numbers introduced. A good indication of this impracticality is that even for the simple generic example of Section 7 , an exact parametric form output by Levin's algorithm fills up roughly 20 megabytes of space! Overall, Levin's method is thus largely impractical. This fact has again recently been noted in [10], where an algorithm for computing a cell in an arrangement of quadrics using resultants is presented.

The key to counter this widely spread pessimism in generalpurpose methods is to improve the pencil method by stripping it of most of its sources of radicals. We show in this paper how this can be done and how the original approach can be turned into a robust, exact and practical method. Using a combination of projective formalism, reduction of quadratic forms and new results characterizing the intersection of quadrics, we show how to avoid the appearance of nested radicals (high-degree algebraic numbers) and how to obtain a parametric representation of the intersection that is near-optimal in the number and depth of radicals involved. Near-optimality in this context roughly means that the parameterization is either fully optimal in the number of radicals or has one possibly extra square root.

\subsection{Our results}

We present an algorithm that computes an exact parametric form of the intersection of two real quadrics in threedimensional space given by implicit equations with rational coefficients. We improve Levin's algorithm in the two following ways. The functions parameterizing the intersection contain no square root whenever it is possible and the coefficients of these functions are algebraic numbers with at most one extra square root. Furthermore, for each geometric type of intersection, the number of square roots in the coefficients is always minimal in the worst case.

These results are summarized in Table 1 and formalized in the following theorem (standard definitions are recalled

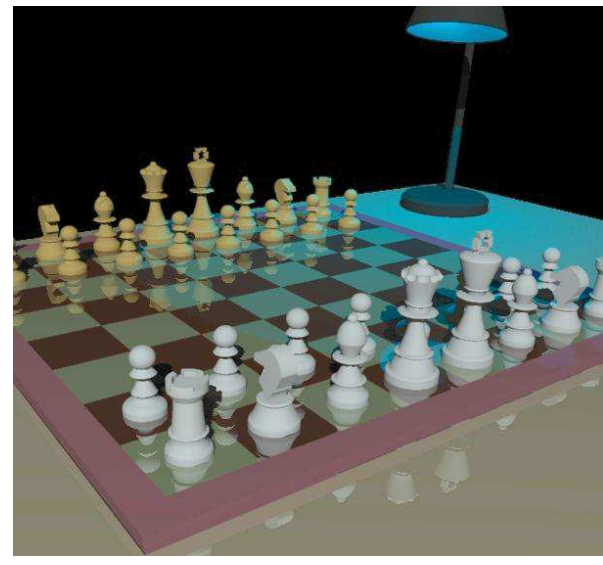

Figure 1: A chess set modeled with quadrics.

in Section 2).

THEOREM 1. In $\mathbb{R}^{3}$ or $\mathbb{P}^{3}$, given two quadrics by their implicit equations whose coefficients are rational, the algorithm of this paper computes a parameterization of their intersection such that each coordinate in projective space is polynomial over an extension field $\mathbb{K}$ of $\mathbb{Q}$ if such a parameterization exists, or is a polynomial in $\mathbb{K}[\xi, \sqrt{\Delta}]$, where $\xi$ is the (real) parameter and $\Delta \in \mathbb{K}[\xi]$ is a polynomial in $\xi$.

In both cases, the parameterization is either optimal in the degree of the field extension $\mathbb{K}$ needed to represent its coefficients or may involve one (and only one) possibly unnecessary square root. In the latter situation, testing for optimality (i.e., determining whether the extra square root is necessary or not) and finding an optimal parameterization are equivalent to finding rational points on a conic or a degree-eight surface.

More concretely our algorithm outputs a parameterization such that each coordinate in projective space is polynomial, if such a parameterization exists, or otherwise is of the form $\alpha+\beta \sqrt{\Delta}$ where $\alpha, \beta$ and $\Delta$ are univariate polynomials (of degree 3, 1 and 4, respectively); see for instance Eq. (7) in Section 7 . The square root $\sqrt{\Delta}$ appears in the parameterization only when no polynomial parameterization exists, that is when the intersection is a smooth quartic, the generic case.

The coefficients of the polynomials are, in the generic case, in (i) an extension of $\mathbb{Q}$ by one square root and, in the nongeneric cases, in (ii) $\mathbb{Q}$ or (iii) an extension of $\mathbb{Q}$ by two square roots or (iv) in some more complicated extension of $\mathbb{Q}$ (see Table 1). The number and depth of radicals involved in the coefficients are always optimal except in cases (i) and (iii) where one of the square roots is possibly unnecessary. Moreover, testing whether one of these square roots is unnecessary and if so finding a parameterization with one less square root is equivalent, in the generic case, to finding a rational point on a degree-eight surface, an outstanding open problem. In the non-generic cases, the test reduces to finding a rational point on a conic, a simpler though difficult task. Thus, the parameterizations output by our algorithm are the best, in terms of number and depth of radicals involved, which may be obtained with easy computation.

We have implemented our algorithm in MuPAD [21]. The code is used in the quadric intersection server available at www.loria.fr/equipes/isa/quadrics.html. We tested it 


\begin{tabular}{|c|c|c|c|c|}
\hline real type of $C$ & generic characterization & typical realization & $\begin{array}{l}\text { worst case format } \\
\text { of parameterization }\end{array}$ & optimality test \\
\hline smooth quartic & no multiple root & 2 ellipsoids & $\begin{array}{c}\mathbb{Q}(\sqrt{\delta})[\xi, \sqrt{\Delta}], \\
\Delta \in \mathbb{Q}(\sqrt{\delta})[\xi], \operatorname{deg} 4\end{array}$ & $\begin{array}{l}\text { rational point on } \\
\text { a deg } 8 \text { surface }\end{array}$ \\
\hline singular quartic & 1 double root (rank 3) & $\begin{array}{l}2 \text { ellipsoids tangent } \\
\text { at one point }\end{array}$ & $\mathbb{Q}(\sqrt{\delta})[\xi]$ & $\begin{array}{l}\text { rational point } \\
\text { on a conic }\end{array}$ \\
\hline cubic and line & 2 double roots (ranks 3 and 3 ) & $\begin{array}{l}2 \text { cones having } \\
\text { a common line }\end{array}$ & $\mathbb{Q}[\xi]$ & optimal \\
\hline 2 conics & 1 double root (rank 2) & ellipsoid and 2 planes & $\mathbb{Q}\left(\sqrt{\delta}, \sqrt{\delta^{\prime}}\right)[\xi]$ & $\begin{array}{l}\text { rational point } \\
\text { on a conic }\end{array}$ \\
\hline conic and 2 lines & 2 double roots (ranks 2 and 3 ) & $\begin{array}{l}\text { cone and } 2 \text { planes, one plane } \\
\text { through the vertex of the cone }\end{array}$ & $\mathbb{Q}(\sqrt{\delta})[\xi]$ & optimal \\
\hline 4 lines & 2 double roots (ranks 2 and 2 ) & 2 pairs of planes & $\begin{array}{c}\mathbb{K}[\xi], \\
\text { degree }(\mathbb{K})=8\end{array}$ & optimal \\
\hline conic and point & 2 double roots (ranks 2 and 3 ) & $\begin{array}{l}\text { ellipsoid and } 2 \text { planes, } \\
\text { with one plane tangent }\end{array}$ & $\mathbb{Q}(\sqrt{\delta})[\xi]$ & $\begin{array}{l}\text { rational point } \\
\text { on a conic }\end{array}$ \\
\hline conic & one double root (rank 2) & $\begin{array}{c}\text { ellipsoid and } 2 \text { planes, } \\
\text { only one plane intersecting }\end{array}$ & $\mathbb{Q}\left(\sqrt{\delta}, \sqrt{\delta^{\prime}}\right)[\xi]$ & $\begin{array}{l}\text { rational point } \\
\text { on a conic }\end{array}$ \\
\hline conic and line & $\begin{array}{c}\mathcal{D}(\lambda) \equiv 0 \quad \& \\
\operatorname{Sing}\left(Q_{S}\right) \cap \operatorname{Sing}\left(Q_{T}\right)=\emptyset\end{array}$ & $\begin{array}{l}\text { cylinder and } 2 \text { planes, one plane } \\
\text { tangent, the other not tangent }\end{array}$ & $\mathbb{Q}[\xi]$ & optimal \\
\hline 4 concurrent lines & $\begin{array}{c}\mathcal{D}(\lambda) \equiv 0 \quad \& \\
\operatorname{Sing}\left(Q_{S}\right) \cap \operatorname{Sing}\left(Q_{T}\right) \neq \emptyset\end{array}$ & $\begin{array}{c}2 \text { cones having } \\
\text { the same vertex }\end{array}$ & $\begin{array}{c}\mathbb{K}[\xi] \\
\text { degree }(\mathbb{K})=24\end{array}$ & optimal \\
\hline
\end{tabular}

Table 1: Snapshot summary of the results of this paper. For each type of intersection, the table gives the corresponding number and multiplicity of multiple roots of $\mathcal{D}(\lambda)$ and rank of the associated quadrics, a typical situation in which this type of intersection occurs, the format of the parameterization obtained by our algorithm in the worst case and the test required to check whether one square root can be avoided.

on "real" scenes such as the chess set (see Figure 1).

Note that a preliminary version of this paper appeared in [6], in which we introduced some of the tools that are used in this paper and sketched a first improvement to Levin's quadric intersection method.

The paper is organized as follows. In Section 2, we present basic definitions, notation, and useful known results. Section 3 summarizes the ideas on which the pencil method of Levin for intersecting quadrics is based and discusses its introduction of nested radicals. In Section 4, we present the general form of our algorithm. In Section 6, this basic algorithm is improved and Theorem 1 is proved. Finally, Section 7 gives one example of an intersection curve computed by our algorithm, before concluding.

\section{NOTATION AND PRELIMINARIES}

In what follows, all the matrices considered are real square matrices. Given a real symmetric matrix $S$ of size $n$, the upper left submatrix of size $n-1$, denoted $S_{u}$, is called the principal submatrix of $S$ and the determinant of $S_{u}$ the principal subdeterminant of $S$.

Formally, we call quadric associated to $S$ the set

$$
Q_{S}=\left\{\mathbf{x} \in \mathbb{P}^{n-1} \mid \mathbf{x}^{T} S \mathbf{x}=0\right\},
$$

where $\mathbb{P}^{n-1}=\mathbb{P}(\mathbb{R})^{n-1}$ denotes the real projective space of dimension $n-1$. (Note that every matrix of the form $\alpha S$, where $\alpha \in \mathbb{R} \backslash\{0\}$, represents the same quadric $Q_{S}$.) When the ambient space is $\mathbb{R}^{n-1}$ instead of $\mathbb{P}^{n-1}$, the quadric is simply $Q_{S}$ minus its points at infinity. In this paper, all the quadrics considered have their coefficients (i.e., the entries of the corresponding matrices) in $\mathbb{Q}$.

Given a quadric $Q_{S}$, the determinant of the associated matrix $S$ (which we also call determinant of $Q_{S}$ by abuse of language) is invariant by a change of coordinates, up to a square factor. Thus, if $S$ and $S^{\prime}$ are two matrices representing the same quadric in different frames, the fields $\mathbb{Q}(\sqrt{\operatorname{det} S})$ and $\mathbb{Q}\left(\sqrt{\operatorname{det} S^{\prime}}\right)$ are equal.

The inertia of a matrix $S$ is classically defined as the triple $\sigma_{S}=\left(\sigma^{+}, \sigma^{-}, \sigma^{0}\right)$, where $\sigma^{+}, \sigma^{-}, \sigma^{0}$ are the numbers of positive, negative and zero eigenvalues of $S$, respec- tively. Since $Q_{S}$ and $Q_{-S}$ represent the same quadric and $\sigma^{0}=n-\sigma^{+}-\sigma^{-}$, we shall instead, by a slight abuse of notation, define the inertia of $S$ and $Q_{S}$ to be $\sigma_{S}=$ $\left(\max \left(\sigma^{+}, \sigma^{-}\right), \min \left(\sigma^{+}, \sigma^{-}\right)\right)$.

In projective space $\mathbb{P}^{3}$, quadrics are characterized by their inertia since two quadrics with the same inertia are projectively equivalent by Sylvester's Inertia Law [17]. In $\mathbb{R}^{3}$ they are characterized by their inertia and the inertia of their principal submatrix. We recall in Table 2 the characterization of Euclidean quadrics in $\mathbb{R}^{3}$.

In $\mathbb{P}^{3}$ or $\mathbb{R}^{3}$, the ruled quadrics are the quadrics surfaces (i.e., the quadrics that are not reduced to a line, a point or the empty set) whose inertia is not $(3,1)$ (see Table 2). Furthermore, a quadric has inertia $(3,1)$ if and only if its determinant is negative.

Let $S$ and $T$ be two real symmetric matrices of the same size. The set of matrices

$$
R(\lambda, \mu)=\left\{\lambda S+\mu T \mid(\lambda, \mu) \in \mathbb{P}^{1}\right\}
$$

is called the pencil of matrices generated by $S$ and $T$. The associated set of quadrics $Q_{R(\lambda, \mu)}$ is called the pencil generated by $Q_{S}$ and $Q_{T}$. The binary form $\mathcal{D}(\lambda, \mu)=\operatorname{det}(R(\lambda, \mu))$ is called the discriminant of the pencil. For the sake of simplicity, we shall simply write that the pencil generated by $S$ and $T$ is $R(\lambda)=\lambda S-T, \lambda \in \overline{\mathbb{R}}=\mathbb{R} \cup\{\infty\}$, where $R(\infty)=S$. Recall that the intersection of any two quadric $Q_{R\left(\lambda_{1}\right)}$ and $Q_{R\left(\lambda_{2}\right)}$ in the pencil is independent of the choice of $\lambda_{1} \neq \lambda_{2}$.

If $\eta_{1}, \ldots, \eta_{m}$ denote $m$ algebraic numbers, the field extension $\mathbb{Q}\left(\eta_{1}, \ldots, \eta_{m}\right)$ is the smallest field containing $\mathbb{Q}$ and $\eta_{1}, \ldots, \eta_{m}$. The degree of a field extension of $\mathbb{Q}$ is the dimension of the extension as a vector space over $\mathbb{Q}$. For instance, the degree of $\mathbb{Q}(\sqrt{a}, \sqrt{b})$, assuming $a, b \in \mathbb{Q}$ and $\sqrt{a}, \sqrt{b}, \sqrt{a b} \notin \mathbb{Q}$, is 4 because $\forall x \in \mathbb{Q}(\sqrt{a}, \sqrt{b})$, there exists $\alpha_{1}, \alpha_{2}, \alpha_{3}, \alpha_{4} \in \mathbb{Q}$ such that $x=\alpha_{1}+\alpha_{2} \sqrt{a}+\alpha_{3} \sqrt{b}+\alpha_{4} \sqrt{a b}$.

If $\xi$ denotes a variable, $\mathbb{Q}[\xi]$ is the ring of polynomials in the variable $\xi$ with coefficients in $\mathbb{Q}$. Similarly, $\mathbb{Q}(\sqrt{\delta})[\xi, \sqrt{\Delta}]$ denotes the ring of polynomials in the variables $\xi$ and $\sqrt{\Delta}$ with coefficients in $\mathbb{Q}(\sqrt{\delta})$.

In the following, a parameterization is called rational over a field $\mathbb{K}$ if and only if the coordinates of the parameter- 


\begin{tabular}{|c|c|c|c|}
\hline Inertia of $S$ & Inertia of $S_{u}$ & Affine reduced canonical form & Euclidean type of $Q_{S}$ \\
\hline \hline$(4,0)$ & $(3,0)$ & $x^{2}+y^{2}+z^{2}+1$ & $\emptyset$ (imaginary ellipsoid) \\
\hline$(3,1)$ & $(3,0),(2,1)$, & $x^{2}+y^{2}+z^{2}-1, x^{2}+y^{2}-z^{2}+1$, & $\begin{array}{c}\text { ellipsoid, hyperboloid of two sheets, } \\
\text { elliptic paraboloid }\end{array}$ \\
& $(2,0)$ & $x^{2}+y^{2}+z$ & point, $\emptyset$ (imaginary elliptic cylinder) \\
\hline$(3,0)$ & $(3,0),(2,0)$ & $x^{2}+y^{2}+z^{2}, x^{2}+y^{2}+1$ & $\begin{array}{c}\text { cone, elliptic cylinder, } \\
(2,2)\end{array}$ \\
\hline$(2,1)$ & $(2,1),(1,1),(2,0)$, & $x^{2}+y^{2}-z^{2}-1, x^{2}-y^{2}+z$ & hyperboloid of one sheet, hyperbolic paraboloid \\
& $(1,1),(1,0)$ & $x^{2}+y^{2}-z^{2}, x^{2}+y^{2}-1$ & hyperbolic cylinder, parabolic cylinder \\
\hline$(2,0)$ & $(2,0),(1,0)$ & $x^{2}-y^{2}+1, x^{2}+y$ & line, $\emptyset$ (imaginary parallel planes) \\
\hline$(1,1)$ & $(1,1),(1,0),(0,0)$ & $x^{2}+y^{2}, x^{2}+1$ & intersecting planes, parallel planes, simple plane \\
\hline$(1,0)$ & $(1,0),(0,0)$ & $x^{2}-y^{2}, x^{2}-1, x$ & double plane, $\emptyset$ \\
\hline
\end{tabular}

Table 2: Euclidean type of a quadric $Q_{S}$ in terms of the inertia of $S$ and the corresponding possible inertias of $S_{u}$.

ization are rational functions with coefficients in $\mathbb{K}$, or in projective space, if the coordinates are polynomials with coefficients in $\mathbb{K}$. A curve is called rational over $\mathbb{K}$ if and only if it admits a rational parameterization over $\mathbb{K}$. When $\mathbb{K}=\mathbb{Q}$ the parameterization or curve is simply called rational.

\section{LEVIN'S PENCIL METHOD}

Since our solution to quadric surface intersection builds upon the pencil method of J. Levin, we start by saying a word on the algorithm described in $[18,19]$ for computing a parameterized representation of the intersection of two distinct implicit quadrics $Q_{S}$ and $Q_{T}$ of $\mathbb{R}^{3}$ and on the complexity of its output.

The high-level idea behind this algorithm is: if (say) $Q_{S}$ is ruled, then $Q_{S}$ admits a parameterization which is linear in one of its parameters and plugging this parameterization in the implicit equation of $Q_{T}$ yields a degree-two equation in one of the parameters (instead of a degree-four equation) which can be easily solved to get a parametric representation of $Q_{S} \cap Q_{T}$.

When neither $Q_{S}$ nor $Q_{T}$ are ruled, then one can find a ruled quadric $Q_{R}$ in the pencil generated by $Q_{S}$ and $Q_{T}$, and we are back to the previous case replacing $Q_{S}$ by $Q_{R}$. Levin's key result proves the existence of such a ruled quadric $Q_{R}$ among at most three quadrics in the pencil. These quadrics are such that their associated matrices $\lambda S+\mu T$ have a vanishing principal subdeterminant, or equivalently that $(\lambda, \mu)$ is solution of the homogeneous degree-three equation $\operatorname{det}\left(\lambda S_{u}+\mu T_{u}\right)=0$. The ruled quadrics among these at most three quadrics are called simple ruled quadrics.

TheOREM 2 ([18]). The pencil generated by any two distinct quadrics contains at least one simple ruled quadric.

Though very general, Levin's algorithm is far from being ideal since it introduces non-rational numbers at several different places (leaving aside the fact that it may fail in degenerate situations). Indeed, nested radicals of depth 2 appear when solving the degree-three equation for finding a simple ruled quadric. Then a canonical form of the simple ruled quadric is computed using an eigenvalues/eigenvectors approach, leading to nested radicals of depth 4 . Finally, parameterizing the simple ruled quadric in canonical form may induce nested radicals of depth 5 .

Hilbert's Irreducibility Theorem implies that the degreethree equation above has no rational root generically and that nesting depth 5 is actually the generic situation. Thus, the coefficients of the parameterization of $Q_{S} \cap Q_{T}$ involve radicals of nesting depth 5 for almost all choices of input quadrics $Q_{S}$ and $Q_{T}$. In addition, the degree of the field extension on which these coefficients are defined may be huge, making the output almost impossible to deal with. The example we consider in Section 7 is a good indication of this impracticality.

Thus the original pencil method can easily suffer from precision and robustness problems. If a floating point representation of numbers is used, the result may be wrong (geometrically and topologically) or, worse, the program may crash. Even exact arithmetic seems out of reach because of the high degree of the algebraic numbers involved.

Note finally that Levin's algorithm outputs non-rational parameterizations even when one exists. Further use of the output may thus be unnecessarily complicated.

\section{OUR METHOD}

We now present our solution to the intersection of implicit quadrics. Our method, which is both robust and practical, removes most of the sources of radicals in Levin's algorithm, in such a way that the output is near-optimal.

We start by giving the key ingredients and stating the main theorem on which our approach is based. We then outline our basic algorithm, after which we detail the steps of the method. In Section 6, we show that, in the generic case (i.e., when the discriminant of the pencil has no multiple root), this basic algorithm produces a parameterization of the intersection that is near-optimal in the number of radicals involved. In the other cases, we either prove that this basic is optimal or near-optimal, or show how to turn our intersection algorithm into a near-optimal one.

\subsection{Key ingredients}

The first ingredient of our approach is to work not just over $\mathbb{R}^{3}$ but over the real projective space $\mathbb{P}^{3}$. Apart from reducing the number of different cases to examine (see Table 2), working in projective space has a deep impact on the method.

In our algorithm, quadrics of inertia different from $(3,1)$ (i.e., ruled quadrics) play the role of simple ruled quadrics in Levin's method. To pursue this parallel further, we need two things. First, we give in Table 3, for all projective quadrics of inertia other than $(3,1)$, parameterizations that are linear in one of their parameters and optimal in the number of radicals (see Theorem 7). That these parameterizations are proper parameterizations of the projective quadrics (i.e., there is a one-to-one correspondence between the points of the quadric and the parameters) is straightforward and left 


\begin{tabular}{|c|c|c|}
\hline inertia of $S$ & canonical equation $(a, b, c, d>0)$ & parameterization $\mathbf{X}=[x, y, z, w]$ \\
\hline \hline$(4,0)$ & $a x^{2}+b y^{2}+c z^{2}+d w^{2}=0$ & $Q_{S}=\emptyset$ \\
\hline$(3,0)$ & $a x^{2}+b y^{2}+c z^{2}=0$ & $Q_{S}$ is a point \\
\hline$(2,2)$ & $a x^{2}+b y^{2}-c z^{2}-d w^{2}=0$ & $\mathbf{X}=\left[\frac{u t+a v s}{a}, \frac{u s-b v t}{b}, \frac{u t-a v s}{\sqrt{a c}}, \frac{u s+b v t}{\sqrt{b d}}\right],(u, v),(s, t) \in \mathbb{P}^{1}$ \\
\hline$(2,1)$ & $a x^{2}+b y^{2}-c z^{2}=0$ & $\mathbf{X}=\left[u v, \frac{u^{2}-a b v^{2}}{2 b}, \frac{u^{2}+a b v^{2}}{2 \sqrt{b c}}, s\right],(u, v, s) \in \mathbb{P}^{\star 2}$ \\
\hline$(2,0)$ & $a x^{2}+b y^{2}=0$ & $\mathbf{X}=[0,0, u, v],(u, v) \in \mathbb{P}^{1}$ \\
\hline$(1,1)$ & $a x^{2}-b y^{2}=0$ & $\mathbf{X}_{1}=\left[u, \frac{\sqrt{a b}}{b} u, v, s\right], \mathbf{X}_{2}=\left[u,-\frac{\sqrt{a b}}{b} u, v, s\right],(u, v, s) \in \mathbb{P}^{2}$ \\
\hline$(1,0)$ & $a x^{2}=0$ & $\mathbf{X}=[0, u, v, s],(u, v, s) \in \mathbb{P}^{2}$ \\
\hline
\end{tabular}

Table 3: Optimal parameterizations of projective quadrics of inertia different from (3,1). (For the case $(2,1), \mathbb{P}^{\star 2}$ denotes the real quasi-projective space defined as the quotient of $\mathbb{R}^{3} \backslash\{0,0,0\}$ by the equivalence relation $\sim$ where $(u, v, s) \sim\left(u^{\prime}, v^{\prime}, s^{\prime}\right)$ iff $\exists \lambda \in \mathbb{R} \backslash\{0\}$ such that $(u, v, s)=\left(\lambda u^{\prime}, \lambda v^{\prime}, \lambda^{2} s^{\prime}\right)$.)

out of the paper. Second, we need something similar to Levin's Theorem 2 in the projective setting. We prove, in Section 5 , the following much stronger result (note that the first part of the theorem follows from Theorem 2).

THEOREM 3. The pencil generated by any two distinct quadrics contains at least one quadric of inertia different from $(3,1)$. If no such quadric has rational coefficients, then the intersection of the two initial quadrics is reduced to two distinct points.

This theorem is critical for our algorithm. Indeed, it ensures that the two quadrics we actually intersect have rational coefficients, except in one very specific situation where the two quadrics intersect in only two points. This means that we remove the first source of nested radicals in Levin's algorithm.

Another basic ingredient of our approach is the use of Gauss' method for the reduction of quadratic forms for computing a canonical form of a ruled projective quadric $Q_{R}$, instead of the eigenvalues/eigenvectors approach used by Levin. Since Gauss' reduction is rational (the elements of the matrix $P$ such that $P^{T} R P$ is diagonal are rational), this removes two other layers of nested radicals from Levin's algorithm.

We also provide parameterization of ruled quadrics that are worst-case optimal in some sense (see Theorem 7). This decreases the number of different radicals in the coefficients of the parameterization and thus the degree of the field extension in which the coefficients live.

The last basic ingredient is a case-by-case analysis of the different types of intersection that may occur and, for each case where our basic algorithm is not near-optimal, we show how to turn it into an optimal or near-optimal one.

\subsection{Algorithm outline}

Armed with these ingredients, we are now in a position to outline our method. Let $Q_{R(\lambda)}, R(\lambda)=\lambda S-T$, be the pencil generated by the quadrics $Q_{S}$ and $Q_{T}$ of $\mathbb{P}^{3}$. The outline of our intersection algorithm is as follows (details of the first three steps follow in ensuing sections).

1. Find a quadric $Q_{R}$ with rational coefficients in the pencil, such that $\operatorname{det} R>0$ if possible or $\operatorname{det} R=0$ otherwise. (If no such $R$ is found, the intersection is reduced to two points, which we output.) If the inertia of $R$ is $(4,0)$, output empty intersection. Otherwise, proceed.

Assume for the sake of simplicity that $Q_{R} \neq Q_{S}$; then $Q_{S} \cap Q_{R}=Q_{S} \cap Q_{T}$.
2. If the inertia of $R$ is not $(2,2)$, apply Gauss' reduction of quadratic forms to $R$.

If the inertia of $R$ is $(2,2)$, find a rational point close enough to $Q_{R}$ such that the quadric $Q_{R^{\prime}}$ through this point in the pencil has inertia $(2,2)$. Rename $Q_{R^{\prime}}$ by $Q_{R}$. Compute a frame in which $P^{T} R P$ is the diagonal matrix $\operatorname{diag}(1,1,-1,-\delta)$, with $\delta \in \mathbb{Q}$.

In the local frame, $Q_{R}$ is described by one of the parameterizations $\mathbf{X}$ of Table 3. Compute the parameterization $\mathbf{X}_{R}=P \mathbf{X}$ of $Q_{R}$ in the initial frame.

3. Consider the equation

$$
\mathbf{X}_{R}^{T} S \mathbf{X}_{R}=0
$$

of degree two in one of the parameters. Solve it in terms of this parameter and compute the domain of the solution. Then substitute this parameter as a function of the other in $\mathbf{X}_{R}$ to obtain a parameterization of the intersection of $Q_{S}$ and $Q_{T}$.

\subsection{Details of Step 1}

The detailed description of Step 1 is as follows. Let $\mathcal{D}(\lambda)=$ $\operatorname{det}(R(\lambda))$.

1. a. If $\mathcal{D}(\lambda) \equiv 0$, set $R=T$ and proceed.

b. Otherwise, compute isolating intervals for the real roots of $\mathcal{D}(\lambda)$ (using for instance the algorithm described in $[22])$. If $\mathcal{D}(\lambda)$ has no real root, set $R=T$ and proceed. Compute a rational number in between each of the separating intervals and compute the inertia of the corresponding quadrics. If one of the inertias is $(4,0)$, then output $Q_{S} \cap Q_{T}=\emptyset$. Otherwise, if one of these inertias is $(2,2)$, proceed with the corresponding quadric.

c. Otherwise (if $\mathcal{D}(\lambda) \not \equiv 0$ and $\mathcal{D}(\lambda) \leqslant 0$ ), compute the greatest common divisor $\operatorname{gcd}(\lambda)$ of $\mathcal{D}(\lambda)$ and its derivative with respect to $\lambda$. If $\operatorname{gcd}(\lambda)$ has a rational root $\lambda_{0}$, proceed with the corresponding quadric $Q_{R\left(\lambda_{0}\right)}$. Otherwise, compute the two points of $Q_{S} \cap Q_{T}$.

To assert the correctness of this algorithm, we have several things to prove. First, we make clear why, when looking for a quadric in the pencil with inertia different from those of $Q_{S}$ and $Q_{T}$, the right polynomial to consider is $\mathcal{D}(\lambda)$.

Lemma 4. The inertia of $R(\lambda)$ is invariant on any interval of $\lambda$ not containing a root of $\mathcal{D}(\lambda)$.

Proof. It suffices to realize that the eigenvalues of $R(\lambda)$ are continuous functions of $\lambda$ and that the characteristic polynomial of $R(\lambda)$, i.e. $\operatorname{det}(R(\lambda)-l I)$, is a polynomial in 
$l$ whose constant coefficient is $\mathcal{D}(\lambda)$, where $I$ is the identity matrix of size 4 .

A quadric of inertia $(4,0)$ is empty of real points (see Table 2). Thus if such a quadric is found in the pencil (in Step 1.b), $Q_{S}$ and $Q_{T}$ have an empty (real) intersection. It is interesting to note that the converse is also true, i.e., emptiness of the intersection implies the presence of a definite matrix in the pencil, which allows us to state the following proposition.

Proposition 5. If $Q_{S} \cap Q_{T}=\emptyset$, Step 1 of our algorithm detects it and outputs empty intersection.

Proof. This proposition is a direct consequence of Lemma 4 and a theorem in linear algebra, originally proved in 1936/1937 by Finsler [8], which states that, for any $n \geqslant 3$ and any pair $S, T$ of real symmetric $n \times n$ matrices, the intersection of $Q_{S}$ and $Q_{T}$ is empty if and only if there exists a matrix $R$ of inertia $(n, 0)$ in the pencil generated by $S$ and $T$.

Finally, note that, in Step 1.c, if $\operatorname{gcd}(\lambda)$ does not admit a rational root, then $Q_{S}$ and $Q_{T}$ intersect in two points. Indeed, since no quadric of positive determinant has been found in Step 1.b, there exists no quadric in the pencil with rational coefficients and inertia different from $(3,1)$. Therefore, by Theorem $3, Q_{S}$ and $Q_{T}$ intersect in two points. We do not describe here how to compute these two points because of the lack of space (see the proof of Theorem 3 for more insight on the question).

\subsection{Details of Step 2}

Let $\sigma_{R}$ denote the inertia of $R$.

\subsubsection{When $\sigma_{R} \neq(\mathbf{2}, 2)$}

When the inertia of $R$ is different from $(2,2)$, we use Gauss' reduction of quadratic forms and parameterize the resulting quadric, whose associated matrix $P^{T} R P$ is diagonal. In view of Sylvester's Inertia Law, the reduced quadric $Q_{P^{T} R P}$ has the same inertia as $R$. Thus $Q_{P^{T} R P}$ can be parameterized with at most one square root by one of the parameterizations $\mathbf{X}$ of Table 3. Since Gauss' reduction is rational (i.e., the coefficients of $P$ are rational), the parameterization $P \mathbf{X}$ of $Q_{R}$ contains at most one square root.

\subsubsection{When $\sigma_{R}=(2,2)$}

According to Theorem 7 (Section 6.1), the parameterization of $Q_{R}$ of Table 3 is optimal in the number of radicals if $Q_{R}$ has no rational point (which can happen). Thus, in the worst case, its coefficients may live in an extension $\mathbb{Q}(\sqrt{m}, \sqrt{n})$ of degree 4 of $\mathbb{Q}$. We show here that there exists, in the neighborhood of $Q_{R}$, a quadric $Q_{R^{\prime}}$ with rational coefficients such that

$$
Q_{S} \cap Q_{R^{\prime}}=Q_{S} \cap Q_{R}=Q_{S} \cap Q_{T}
$$

and the coefficients of the parameterization of $Q_{R^{\prime}}$ are in $\mathbb{Q}\left(\sqrt{\operatorname{det} R^{\prime}}\right)$.

Compute an arbitrary point $\mathbf{p} \in \mathbb{P}^{3}(\mathbb{R})$ on $Q_{R}$ (for instance by using the parameterization of $Q_{R}$ ). If $\mathbf{p}$ is rational then take $Q_{R^{\prime}}=Q_{R}$ and move on to the next paragraph. Otherwise, approximate $\mathbf{p}$ by a point $\mathbf{p}^{\prime} \in \mathbb{P}^{3}(\mathbb{Q})$. A simple procedure enables us to choose $\mathbf{p}^{\prime}$ not on $Q_{S} \cap Q_{T}$. Then $\mathbf{p}^{\prime}$ belongs to a unique quadric $Q_{R^{\prime}}$ in the pencil defined by

$$
R^{\prime}=\frac{Q_{T}(\mathbf{p})}{Q_{S}(\mathbf{p})} S-T
$$

where $Q_{S}(\mathbf{p}), Q_{T}(\mathbf{p})$ denote the values of $Q_{S}$ and $Q_{T}$ at point $\mathbf{p}$ (i.e., $\mathbf{p}^{T} S \mathbf{p}$ and $\left.\mathbf{p}^{T} T \mathbf{p}\right)$. Note that the parameters $\lambda_{0}$ and $\lambda_{0}^{\prime}$ such that $R=R\left(\lambda_{0}\right)$ and $R^{\prime}=R\left(\lambda_{0}^{\prime}\right)$ get arbitrarily close to one another as $\mathbf{p}^{\prime}$ gets close to $\mathbf{p}$. Thus if $\mathbf{p}^{\prime}$ is close enough to $\mathbf{p}, R^{\prime}$ has the same inertia $(2,2)$ as $R$, by Lemma 4; otherwise, refine the approximation of $\mathbf{p}$.

We now have a quadric $Q_{R^{\prime}}$ of inertia $(2,2)$ and a rational point on $Q_{R^{\prime}}$. Consider any rational line through $\mathbf{p}^{\prime}$ that is not in the plane tangent to $Q_{R^{\prime}}$ at $\mathbf{p}^{\prime}$. This line further intersects $Q_{R^{\prime}}$ in another rational point $\mathbf{p}^{\prime \prime}$. Compute the rational transformation $P$ sending $\mathbf{p}^{\prime}, \mathbf{p}^{\prime \prime}$ onto $(1, \pm 1,0,0)$. Apply this transformation to $R^{\prime}$ and then apply Gauss' reduction of quadratic forms. In the local frame, $Q_{R^{\prime}}$ has equation (up to a constant factor)

$$
x^{2}-y^{2}+\alpha z^{2}+\beta w^{2}=0,
$$

with $\alpha \beta<0$. Now consider the linear transformation whose matrix is $P^{\prime}$

$$
P^{\prime}=\frac{1}{2}\left(\begin{array}{cccc}
1+\alpha & 0 & 1-\alpha & 0 \\
1-\alpha & 0 & 1+\alpha & 0 \\
0 & 2 & 0 & 0 \\
0 & 0 & 0 & 2 \alpha
\end{array}\right) .
$$

Applying $P^{\prime}$ to the already reduced quadric of Eq. (2) gives the equation

$$
x^{2}+y^{2}-z^{2}-\delta w^{2}=0,
$$

where $\delta=-\alpha \beta>0$. The quadric of Eq. (3) can be parameterized by

$$
\mathbf{X}=\left(u t+v s, u s-v t, u t-v s, \frac{u s+v t}{\sqrt{\delta}}\right),
$$

with $(u, v),(s, t) \in \mathbb{P}^{1}$ (see Table 3 ).

The three consecutive transformation matrices have rational coefficients thus $\mathbb{Q}(\sqrt{\delta})=\mathbb{Q}\left(\sqrt{\operatorname{det} R^{\prime}}\right)$ and the product of these transformation matrices with $\mathbf{X}$ is a polynomial parameterization of $Q_{R^{\prime}}$ with coefficients in $\mathbb{Q}(\sqrt{\delta}), \delta \in \mathbb{Q}$.

\subsection{Details of Step 3}

Solving Eq. (1) can be done as follows. Recall that the content in the variable $x$ of a multivariate polynomial is the gcd of all the coefficients of the $x^{i}$.

Equation (1) may be seen as a quadratic equation in one of the variables. For instance, if $R$ has inertia $(2,2)$, Equation (1) is a homogeneous biquadratic equation in the variables $\xi=(u, v)$ and $\tau=(s, t)$. Using only gcd computations, we can factor it in its content in $\xi$ (which is a polynomial in $\tau$ or a constant), its content in $\tau$ and a remaining factor. If the content in $\xi$ (or in $\tau$ ) is not constant, solve it in $\tau$ (in $\xi$ ); substituting the obtained real values in $\mathbf{X}$, we have a parameterization of some components of $Q_{S} \cap Q_{T}=Q_{S} \cap Q_{R}$ in the frame in which $Q_{R}$ is canonical. If the remaining factor is not constant, solve it in a parameter in which it is linear, if any, or in $\tau$. Substituting the result in $\mathbf{X}$, we have a parameterization of the last component of the intersection. If the equation which is solved is not linear, the domain of the parameterization is the set of $\xi$ such that the degree-four polynomial $\Delta(\xi)=b^{2}(\xi)-4 a(\xi) c(\xi)$ is positive, where $a(\xi), b(\xi)$ and $c(\xi)$ are the coefficients of $\tau^{2}, \tau$ and 1 in Eq. (1), respectively. 


\section{PROOF OF THEOREM 3}

To prove Theorem 3 (Section 4.1), we use the canonical form theorem for pairs of symmetric matrices - see [25, 26], and we state the part of this theory which we need here.

Theorem 6 (CAnonical Pair Form). Let $S$ and $T$ be two real symmetric matrices of size $n$, with $\operatorname{det} S \neq 0$. Then the following properties hold.

(a) The characteristic polynomial of $S^{-1} T$ and the determinant of $\lambda S-T$ have the same roots $\lambda_{j}$ with the same multiplicities $\gamma_{j}$.

(b) The matrices $S$ and $T$ are simultaneously congruent by a real congruence transformation to (or, in other words, there is a projective change of coordinates such that the matrices of the corresponding quadrics are) $S^{\prime}=\operatorname{diag}\left(\varepsilon_{1} E_{1}, \ldots\right.$, $\left.\varepsilon_{m} E_{m}\right)$ and $T^{\prime}=\operatorname{diag}\left(F_{1}, \ldots, F_{m}\right)$, respectively, where $\varepsilon_{i}=$ $\pm 1, E_{i}$ denotes a square matrix of the form

$$
\left(\begin{array}{ll}
0 & 1 \\
& \\
1 & 0
\end{array}\right) \text { or }(1)
$$

and $F_{i}$ is a symmetric matrix of the same size as $E_{i}$.

(c) For each $i$, the matrix $\varepsilon_{i} E_{i}^{-1} F_{i}$ has only one real or two complex conjugate eigenvalues.

(d) The sum of the sizes of the blocks corresponding to one of the $\lambda_{j}$ is the multiplicity $\gamma_{j}$ if $\lambda_{j}$ is real or twice this multiplicity if $\lambda_{j}$ is complex. The number of the corresponding blocks is $\eta_{j}=n-\operatorname{rank}\left(\lambda_{j} S-T\right)$, and $1 \leqslant \eta_{j} \leqslant \gamma_{j}$.

Proof of Theorem 3. Consider a pencil of real symmetric $4 \times 4$ matrices generated by two symmetric matrices $S$ and $T$ of inertia $(3,1)$. We may suppose that $S$ and $T$ have the block diagonal form of the above theorem.

If all the blocks have size 2 or 4 , the determinant of $S$ is equal to $\varepsilon_{1}^{2} \varepsilon_{2}^{2}=1$ or $\varepsilon_{1}^{4}=1$ and thus is positive, contradicting our hypothesis. Thus, there is a block of size 1 and $\operatorname{det}(\lambda S-T)$ has at least one real root. It follows that the matrix of the pencil corresponding to this root has a vanishing determinant and thus an inertia different from $(3,1)$. This proves the first part of the theorem.

If $\operatorname{det}(\lambda S-T)$ has a simple real root, there is an interval of values for $\lambda$ for which $\operatorname{det}(\lambda S-T)>0$ and the matrix corresponding to any rational value of $\lambda$ in this interval has rational coefficients and inertia different from $(3,1)$. If $\operatorname{det}(\lambda S-T)$ has either a double real root and two complex roots, two rational double real roots or a quadruple real root, the quadrics corresponding to the real roots have rational coefficients and have inertia different from $(3,1)$.

Thus we are left with the case where $\operatorname{det}(\lambda S-T)$ has two non-rational double real roots, which are algebraic conjugates. In other words,

$$
\operatorname{det}(\lambda S-T)=c\left(\lambda-\lambda_{1}\right)^{2}\left(\lambda-\lambda_{2}\right)^{2},
$$

with $\lambda_{1}, \lambda_{2} \in \mathbb{R} \backslash \mathbb{Q}$ and $\lambda_{2}=\bar{\lambda}_{1}$ its (real algebraic) conjugate. Following the notation of the Canonical Pair Form Theorem, we have $\gamma_{1}=\gamma_{2}=2$ and $1 \leqslant \eta_{i} \leqslant 2$, for $i=1,2$. In other words, $\left(\eta_{1}, \eta_{2}\right) \in\{(1,1),(1,2),(2,1),(2,2)\}$.

We can quickly get rid of the case $\left(\eta_{1}, \eta_{2}\right)=(1,1)$. Indeed, in this case the blocks have size 2 and $S$ is not of inertia $(3,1)$.

We can also eliminate the cases $\left(\eta_{1}, \eta_{2}\right) \in\{(1,2),(2,1)\}$, because the matrices $\lambda_{1} S-T$ and $\lambda_{2} S-T$ are algebraically conjugate, and so must have the same rank.
We are thus left with the case $\left(\eta_{1}, \eta_{2}\right)=(2,2)$. In this situation, $S$ and $T$ have four blocks, i.e., they are diagonal:

$$
S=\operatorname{diag}\left(\varepsilon_{1}, \varepsilon_{2}, \varepsilon_{3}, \varepsilon_{4}\right), \quad T=\operatorname{diag}\left(\mu_{1}, \mu_{2}, \mu_{3}, \mu_{4}\right) .
$$

Since $\left(\eta_{1}, \eta_{2}\right)=(2,2)$, the two matrices $\lambda_{1} S-T$ and $\lambda_{2} S-T$ have rank 2. Replacing $S$ and $T$ by these matrices, which generate the same pencil, we get that exactly two of the $\varepsilon_{i}$ and two of the $\mu_{i}$ are null. As the pencil contains quadrics of rank 4 , we cannot have $\varepsilon_{i}$ and $\mu_{i}$ simultaneously null. Thus, after permuting the basis elements, we get

$$
S=\operatorname{diag}\left(\varepsilon_{1}, \varepsilon_{2}, 0,0\right) \text { and } T=\operatorname{diag}\left(0,0, \mu_{3}, \mu_{4}\right),
$$

with $\varepsilon_{1} \varepsilon_{2} \mu_{3} \mu_{4} \neq 0$.

There are matrices in the pencil whose determinant is negative (the two initial matrices $S$ and $T$ ). Thus, since $\operatorname{det}(\lambda S-T)$ has two double roots, all the matrices in the pencil except $S$ and $T$ have negative determinant. Hence $\varepsilon_{1} \varepsilon_{2} \mu_{3} \mu_{4}=\operatorname{det}(S+T)<0$ and $\varepsilon_{1} \varepsilon_{2}$ and $\mu_{3} \mu_{4}$ have opposite signs. It follows that one of $S$ and $T$ has inertia $(2,0)$, say $S$, and the other has inertia $(1,1)$. Thus, in projective space, $Q_{S}$ is a straight line which intersects the pair of real planes $Q_{T}$. Since $Q_{S} \cap Q_{T}$ is contained in all the quadrics of the pencil and since the pencil has quadrics of inertia $(3,1)$ (which are not ruled), the line $Q_{S}$ is not included in $Q_{T}$ and the intersection is reduced to two real points. Moreover, these points are necessarily distinct. If they were not, they would lie on the intersection of $Q_{S}$ and on the singular line of $Q_{T}$, which is impossible since the two lines have $x_{1}=x_{2}=0$ and $x_{3}=x_{4}=0$ as projective equations.

Note that a pencil generated by two quadrics of inertia $(3,1)$ may contain no quadric with rational coefficients of inertia different from $(3,1)$. Consider for instance

$$
\begin{gathered}
Q_{S}: 2 x^{2}-2 x z-2 y w+z^{2}+w^{2}=0, \\
Q_{T}: 4 x^{2}+2 y^{2}-2 y w+z^{2}-6 x z+3 w^{2}=0 .
\end{gathered}
$$

Then, $\operatorname{det}(\lambda S-T)=-\left(\lambda^{2}-5\right)^{2}$ and $Q_{ \pm \sqrt{5} S-T}$ are the only quadrics in the pencil with inertia different from $(3,1)$.

\section{OPTIMALITY}

We now focus on optimality issues. We start, in Sections 6.1 , with a theorem on the worst-case optimality (in the number of radicals involved) of the parameterizations of Table 3. Based on this general result, we make in Sections 6.2 and 6.3 a case-by-case analysis of the different types of intersection that may occur and, for each type, we show that the basic algorithm of Section 4 is near-optimal or how it can be modified to obtain a near-optimal parameterization of the intersection curve. Furthermore, we show that the near-optimal parameterization is either fully optimal or that testing whether a square root in the coefficients can be avoided is equivalent to finding a rational point on a curve or a surface. Table 1 summarizes these results and gives, for each case, the optimal or near-optimal form of the parameterization of the intersection and the optimality test, if any.

In the rest of this section, $C$ stands for the intersection of $Q_{S}$ and $Q_{T}$. Due to the lack of space, we omit the proof of many results and merely sketch the proofs of some others. Note in particular that we omit the proofs of the relationship between the geometric type of $C$ and the number of multiple roots of the discriminant of the pencil and the rank of the associated matrices. Note also that we do not consider here 
all the possible cases and that, in the cases we do consider, we focus on the "generic" situations. For instance, when $C$ consists of two conics, the quadric corresponding to the double root of $\mathcal{D}(\lambda)$ is "generically" a pair of planes. A more degenerate situation is when the two planes are in fact a single double plane. In this case, the algorithm given in Section 6.2 .3 can be simplified.

\subsection{Preliminaries}

Theorem 7 states that among the parameterizations linear in one of the parameters, the ones of Table 3 have, in the worst case, an optimal number of radicals, i.e., for each type of projective quadric, there are examples of surfaces with as many square roots as in Table 3 .

Note that although the worst-case optimality of the parameterizations of quadrics does not directly imply the nearoptimality of the output parameterizations of the intersections, the results stated in Theorem 7 are crucial for the proof of Theorem 1.

THEOREM 7. In the set of parameterizations linear in one of the parameters, the parameterizations of Table 3 are worstcase optimal in the degree of the extension of $\mathbb{Q}$ on which they are defined. For a quadric of inertia other than $(2,1)$ and $(2,2)$, the parameterization is optimal in all cases. For a quadric of inertia $(2,1)$ or $(2,2)$, the parameterization is optimal if the quadric has no rational point (over $\mathbb{Q}$ ), which is the case for some quadrics.

If a quadric of inertia $(2,1)$ has a rational point other than the singular point, it has a canonical equation $x^{2}+y^{2}-z^{2}$, for which the corresponding parameterization is rational (and thus optimal). If a quadric of inertia $(2,2)$ has a rational point, it has a canonical equation $x^{2}+y^{2}-z^{2}-d w^{2}$, where $d$ is the determinant of the quadric, for which the corresponding parameterization is optimal in the degree of the extension of $\mathbb{Q}$.

We now state two other preliminary results.

Proposition 8. If the intersection of two given quadrics has a parameterization involving only one square root (in the parameterization or in the coefficients), then there exists a quadric with rational coefficients in the pencil that contains a rational line.

Proposition 9. If a quadric contains a rational line, its determinant is a square in $\mathbb{Q}$.

\subsection{When $R$ has inertia $(2,2)$}

Assume here that the matrix $R$ in Step 2 of our algorithm has inertia $(2,2)$. After Step 2 of our algorithm, $Q_{R}$ has a parameterization on $\mathbb{Q}(\sqrt{\delta})$ that is bilinear in $\xi=(u, v)$ and $\tau=(s, t)$. Substituting in $Q_{S}$ yields an equation $\Omega$ of bidegree $(2,2)$ in $(\xi, \tau)$. After resolution and substitution in $Q_{R}$, we get a parameterization of $C$ in $\mathbb{Q}(\sqrt{\delta})[\xi, \sqrt{\Delta}]$ with $\Delta \in \mathbb{Q}(\sqrt{\delta})[\xi]$ of degree 4 .

Let $C_{\Omega}$ be the curve zero-set of $\Omega$. $C_{\Omega}$ is a curve of bidegree $(2,2)$ in $\mathbb{P}^{1} \times \mathbb{P}^{1}$. We have the following classical result.

Fact (Geometric interpretation). The parameterization of $Q_{R}$ defines an isomorphism between $C$ and the plane curve $C_{\Omega}$ defined by $\Omega$. In particular, $C$ and $C_{\Omega}$ have the same genus, irreducibility and factorization.

Thus, for instance, when $C$ consists of a cubic and a line, $C_{\Omega}$ has a cubic factor of bidegree $(2,1)$ and a linear factor of bidegree $(0,1)$. When $C$ consists of a conic and two lines, $C_{\Omega}$ has a quadratic factor of bidegree $(1,1)$ and two linear factors of bidegree $(1,0)$ and $(0,1)$.

By substituting $v=t=1$ in $\Omega, C_{\Omega}$ can be identified with a curve of degree 4 in $\mathbb{P}^{2}$ having two double points at infinity with distinct tangents (possibly after a projective transformation of $C_{\Omega}$ ). Now, since a curve of $\mathbb{P}^{2}$ of degree $d$ with $b$ simple nodes has genus

$$
g=\frac{1}{2}(d-1)(d-2)-b
$$

$C_{\Omega}$ has genus at most 1 . Thus, the genus of $C$ is 1 when the intersection of $Q_{S}$ and $Q_{T}$ is non-singular, i.e., when $C_{\Omega}$ is smooth, and 0 otherwise. (See [27] for related questions on the classification of the intersection of quadrics.)

In what follows, we examine the possible cases for the real type of $C$, as a function of the number of double real roots of the discriminant of the pencil $\mathcal{D}(\lambda)$ and the rank of the associated quadrics. Determining whether $\mathcal{D}(\lambda)$ has double real roots can be achieved by computing its discriminant $\Gamma(\mathcal{D})$ (see for instance [4]). When $\mathcal{D}(\lambda)$ has a multiple real root $(\Gamma(\mathcal{D})=0)$, we compute the gcd of $\mathcal{D}(\lambda)$ and its derivative with respect to $\lambda$. The roots of the gcd are the multiple real roots of $\mathcal{D}(\lambda)$. We then compute the inertia of the associated quadrics in the pencil and proceed.

Due to the lack of space, we only present here three representative cases and omit the three remaining cases, $C$ is a singular quartic, $C$ is a conic and two lines, and $C$ is four lines.

\subsubsection{C is a smooth quartic}

Here, $\mathcal{D}(\lambda)$ has no multiple root (i.e., $\Gamma(\mathcal{D}) \neq 0$ ). The fact that $C$ has genus 1 in this case implies that it cannot be parameterized by polynomial functions [12], so $\sqrt{\Delta}$ cannot be avoided. The question now is: can $\sqrt{\delta}$ be avoided? The answer is that there are cases in which it cannot be avoided, as we shall see.

If $\sqrt{\delta}$ can be avoided then, by Proposition 8 , there exists in the pencil a quadric $Q$ with rational coefficients and containing a rational line. The rank of $Q$ is at least 3 by Theorem 6 and since $\mathcal{D}(\lambda)$ has no multiple root. Thus $Q$ has inertia $(2,2)$ or $(2,1)$ (see Table 2$)$. Hence the rational line in $Q$ is not singular and $Q$ contains a non-singular rational point. Furthermore, the determinant of $Q$ is a square in $\mathbb{Q}$, by Proposition 9. Therefore, there is in the pencil a quadric of inertia $(2,2)$ or $(2,1)$ with rational coefficients, having a non-singular rational point and whose determinant is a square in $\mathbb{Q}$. Conversely, if such a quadric exists, it has a rational parameterization by Theorem 7 and thus $\sqrt{\delta}$ can be avoided.

It follows that testing if $\sqrt{\delta}$ can be avoided is equivalent to finding a rational point on the surface of degree 8 of equation

$$
z^{2}=\operatorname{det}\left(Q_{T}(x, y, \mathrm{cst}, 1) \cdot S-Q_{S}(x, y, \mathrm{cst}, 1) \cdot T\right),
$$

where $c s t$ is some constant and $Q(x, y$, cst, 1$)$ is the value of the quadric at the point $(x, y, \mathrm{cst}, 1)$. The idea is to consider the whole range of quadrics in the pencil not by varying the pencil parameter $\lambda$ but by looking at the points of space they go through. It suffices then to notice that every quadric of inertia $(2,1)$ or $(2,2)$ is cut by any given plane, so we can restrict our attention to one particular plane, say $z=$ cst, that does not go through the vertex of any cone of the pencil, and consider the quadrics going through the point $(x, y$, cst, 1$)$, which gives (4). 
There are pairs of quadrics whose intersection is a smooth quartic such that Eq. (4) has no rational solution. One example is $Q_{S}: 5 y^{2}+6 x y+2 z^{2}-w^{2}+6 z w=0, Q_{T}$ : $3 x^{2}+y^{2}-z^{2}-w^{2}=0$. (The proof consists in reducing Eq. (4) modulo 4 and 8.)

Thus the parameterization output by the algorithm of Section 4 is worst-case optimal in the number of radicals when the intersection is a smooth quartic (which is the generic case).

\subsection{2 $C$ is a cubic and a line}

In this case, the line of $C$ is necessarily rational (otherwise its conjugate would also be in $C$ ). $Q_{R}$ thus contains a rational line, which means it has a rational parameterization (by Proposition 9 and Theorem 7), and the parameterization of the intersection is in $\mathbb{Q}[\xi]$.

$C$ is obtained through the basic algorithm of Section 4 . The parameterization of the cubic of $C$ is obtained by solving the bidegree $(2,1)$ factor of $\Omega$ for $\tau$ (the variable of the linear term) in terms of $\xi$ and the parameterization of the line of $C$ is obtained by solving the bidegree $(0,1)$ factor.

\subsubsection{C is two conics}

Factoring $C_{\Omega}$ in two curves of bidegree $(1,1)$ can induce nested radicals, so parameterizing $C$ by solving $\Omega$ directly is not a good idea. Instead, we proceed as follows.

The singular quadric corresponding to the double root of $\mathcal{D}(\lambda)$ is a pair of planes, each containing one of the conics. Separate them. Now, the line $\xi=(0,1), \tau \in \mathbb{P}^{1}$ (lifted back to the original space) cuts each conic in one point. Assume for instance that conic $C_{1}$, containing point $\mathbf{p}_{1}$, is in plane $\omega_{1}$. Then $C_{1}$ can be parameterized by the lines of $\omega_{1}$ through $\mathbf{p}_{1}$. Same for $C_{2}$.

This procedure gives a parameterization of $C$ in $\mathbb{Q}(\sqrt{\delta}$, $\left.\sqrt{\delta^{\prime}}\right)$, where $\delta^{\prime}$ is the discriminant of the pair of planes associated to the double root of $\mathcal{D}(\lambda)$ (an invariant up to a square factor) and $\delta$ the discriminant of $Q_{R} \cdot \sqrt{\delta^{\prime}}$ can never be avoided (although it might be rational), thus the parameterization is optimal only in the cases where $\sqrt{\delta}$ cannot be avoided.

The statement in Theorem 7 concerning projective cones (i.e., quadrics of inertia $(2,1)$ ) can be rephrased for nonsingular conics as follows. Consider a conic defined in a $\mathbb{K}$-rational plane, where $\mathbb{K}$ is some extension of $\mathbb{Q}$. If the conic has a point that is rational over $\mathbb{K}$, then it admits a parameterization that is rational over $\mathbb{K}$. Translated in the context where the intersection consists of two conics, it means that the conics have a parameterization that is rational over $\mathbb{Q}\left(\sqrt{\delta^{\prime}}\right)$ if and only if they have a rational point over that extension. Otherwise, they have a parameterization in $\mathbb{Q}\left(\sqrt{\delta}, \sqrt{\delta^{\prime}}\right)$.

Note that the worst-case degree 4 of the extension on which the parameterization is defined can be attained. Consider for instance $Q_{S}: x^{2}-33 w^{2}=0$ and $Q_{T}: y^{2}+z^{2}-$ $3 w^{2}=0$. The intersection $C=Q_{S} \cap Q_{T}$ has no rational point on $\mathbb{Q}(\sqrt{33})$, so $C$ has no rational point on any extension of degree 2 .

\subsection{When $R$ has inertia $\neq(2,2)$}

We now examine the different types of intersection when the pencil contains no quadric of inertia $(2,2)$. Due to the lack of space we omit the cases where $C$ is a conic and a line, $C$ is a conic, and $C$ is four concurrent lines.

\subsection{1 $C$ is a conic and a point}

Here, $\mathcal{D}(\lambda) \leqslant 0$ and $\mathcal{D}(\lambda)$ has two double real roots, one giving a pair of planes, the other a projective cone having its singular point on one of the planes. $C$ consists of a real conic and two complex conjugate lines lying on the cone. The algorithm in this case is as follows.

The point of $C$ can be computed as the (rational) vertex of the cone. The conic lies in one of the rational planes and can be easily parameterized. For instance, one can obtain a point on the conic by intersecting one of the generators of the cone with the plane containing the conic. The parameterization of this conic is then obtained by the lines of the plane through this point.

The double roots of $\mathcal{D}(\lambda)$ are rational since the two singular quadrics are not conjugate. Thus the cone is rational and, since it has its vertex on one of the planes, the two planes are also rational. Thus only one square root is (possibly) needed to parameterize the conic. Testing for optimality amounts to finding a rational point on this conic.

\section{EXAMPLE}

We now detail one example of computing the intersection of two quadrics with our algorithm. Let $Q_{S}$ and $Q_{T}$ be the two surfaces of inertia $(3,1)$ (an ellipsoid and a hyperboloid of two sheets)

$$
\begin{gathered}
Q_{S}: x^{2}-y^{2}+z^{2}-x y-w y+w^{2}=0 \\
Q_{T}: 2 x^{2}+y^{2}+z^{2}-x y-y z+w y=0
\end{gathered}
$$

Then

$$
\mathcal{D}(\lambda)=\operatorname{det}(\lambda S-T)=\frac{1}{4}\left(-6 \lambda^{4}+12 \lambda^{3}+3 \lambda^{2}-6 \lambda-2\right) .
$$

The discriminant $\Gamma(\mathcal{D})$ of $\mathcal{D}(\lambda)$ is negative here, so $\mathcal{D}(\lambda)$ has two simple real roots. Isolating intervals for these roots are $(0,1)$ and $(1,2)$. A quadric of inertia $(2,2)$ is found between the two roots, for instance at $\lambda=1$. So take $R=S-T$. Gauss' method for the reduction of quadratic forms gives the parameterization of $Q_{R}$ in the global frame

$$
\begin{array}{r}
\mathbf{X}_{R}=(\sqrt{2}(t v+8 s u), 2 \sqrt{2}(t u-s v)+8 s u-t v, \\
4(8 s u+2 s v+2 t u-t v), 4(s v+t u)) .
\end{array}
$$

Using this parameterization, pick a random point on $Q_{R}$, for instance for $(u, v)=(1,1)$ and $(s, t)=(1,-1), \mathbf{p}=$ $(7 \sqrt{2} / 36,1 / 4-\sqrt{2} / 9,1,0)$ and approximate it by the rational point $\mathbf{p}^{\prime}=(0,0,1,0)$. It turns out that $\mathbf{p}^{\prime}$ is also on $Q_{R}$, so, since $\sqrt{\operatorname{det} R}=1 / 2 \in \mathbb{Q}, Q_{R}$ has a rational parameterization. We can find it as described in Section 4.4:

$$
\begin{aligned}
\mathbf{X}_{R}=(s u+t v-2 s v, s u-s v-t u+t v, \\
4 s u-6 s v-2 t u, s u-t v),
\end{aligned}
$$

where $(u, v),(s, t) \in \mathbb{P}^{1}$. Computing $\Omega: \mathbf{X}_{R}^{T} S \mathbf{X}_{R}=0$ gives

$$
\tilde{\xi}^{2}\left(15 \tilde{\tau}^{2}-12 \tilde{\tau}+3\right)+\tilde{\xi}\left(-46 \tilde{\tau}^{2}+16 \tilde{\tau}+2\right)+37 \tilde{\tau}^{2}+1=0,
$$

where $\tilde{\xi}=u / v$ and $\tilde{\tau}=s / t$. Since $\mathcal{D}(\lambda)$ has no multiple root, we know that $Q_{S} \cap Q_{T}$ is a smooth quartic and, by the results of Section $6, \Omega$ is irreducible. We can solve it for $\tilde{\xi}$ in terms of $\tilde{\tau}$ or, more precisely, for $\xi=(u, v)$ in terms of $\tau=(s, t)$. Eq. (6) admits real solutions if and only if its discriminant

$$
\Delta(s, t)=-26 s^{4}+76 s^{3} t-108 s^{2} t^{2}+28 s t^{3}-2 t^{4} \geqslant 0,
$$

that is if and only if $(s, t)=(s, 1)$ with $s \in\left[s_{0}, s_{1}\right]$, where $s_{0}$ (resp. $\left.s_{1}\right)$ is the unique root of $\Delta(s, 1)$ in the interval 
$[1 / 8,5 / 32]$ (resp. [5/32,3/16]). For any such $(s, t)$, plugging the solutions of Eq. (6) into Eq. (5) gives the parameterization of the intersection of $Q_{S}$ and $Q_{T}$

$$
\left(\begin{array}{c}
x \\
y \\
z \\
w
\end{array}\right)=\left(\begin{array}{c}
-7 s^{3}+31 s^{2}-19 s+3 \pm s \sqrt{\Delta(s, 1)} \\
8 s^{3}-4 s^{2}-8 s+4 \pm(s-1) \sqrt{\Delta(s, 1)} \\
2 s^{3}-6 s^{2}-6 s+2 \pm 2(2 s-1) \sqrt{\Delta(s, 1)} \\
23 s^{3}-23 s^{2}+11 s-3 \pm s \sqrt{\Delta(s, 1)}
\end{array}\right), \quad s \in\left[s_{0}, s_{1}\right] .
$$

Note that in general the rational point $\mathbf{p}^{\prime}$ approximating $\mathbf{p}$ is not on a quadric whose determinant is a square. Thus, in general, the coefficients of the parameterization are not integers as in Eq. (7) but of the form $q+q^{\prime} \sqrt{\delta}$ where $q, q^{\prime}$ and $\delta$ are rational numbers (or integers).

In the same setting, Levin's algorithm does not fare well at all. In fact, the intersection of $Q_{S}$ and $Q_{T}$ is, from the point of view of radicals, a worst case (though generic!) situation for Levin's algorithm: $\operatorname{det}\left(R_{u}(\lambda)\right)$ has no rational root, the normalized eigenvectors of $R_{u}\left(\lambda_{0}\right)$ involve radicals of nesting depth 4 and the coefficients of the parameterization of the intersection has nested radicals of depth 5. Finding the exact parametric representation of $C$ this way can hardly be done. And, most importantly, the result is almost useless: even if intermediate results are simplified, the output fills up roughly 20 megabytes of space!

\section{CONCLUSION}

In this paper, we have presented a new algorithm for the robust and practical intersection of two arbitrary quadrics. Our approach, inspired by the pencil method of J. Levin, outputs an explicit representation of the intersection that (i) is the best one from the point of view of the number and depth of radicals involved that can easily be computed and (ii) is usable in practice.

We have implemented our algorithm in MuPAD [21]. The code is used in the quadric intersection server available at www.loria.fr/equipes/isa/quadrics.html. We tested it on "real" scenes such as the chess set (see Figure 1). The chess set has been entirely modeled with quadrics by SGDL Systems Inc. and given to us as a CSG model. We computed the boundary representation of this model which involved computing the intersection of all pairs of quadrics defining the chess pieces. Each piece is modeled with between 12 and 25 quadrics. We then rendered the scene using Candela [1].

\section{REFERENCES}

[1] L. Alonso, F. Cuny, S. Petitjean, J.-C. Paul, S. Lazard, and E. Wies. The virtual mesh: A geometric abstraction for efficiently computing radiosity. ACM Transactions on Graphics, 20(3):169-201, 2001.

[2] M. Benouamer, D. Michelucci, and B. Peroche. Error-free boundary evaluation based on a lazy rational arithmetic: a detailed implementation. Computer-Aided Design 26(6):403-416, 1994.

[3] A. Bowyer, J. Berchtold, D. Eisenthal, I. Voiculescu, and K. Wise. Interval methods in geometric modeling. In Proc. of International Conference on Geometric Modeling and Processing, Hong Kong, 2000. Invited presentation.

[4] J. Cremona. Reduction of binary cubic and quartic forms. LMS Journal of Computation and Mathematics, 2:62-92, 1999.

[5] J. Cremona and D. Rusin. Efficient solution of rational conics. Mathematics of Computation, posted on December 18, 2002 (to appear in print).

[6] L. Dupont, D. Lazard, S. Lazard, and S. Petitjean. Towards the robust intersection of implicit quadrics. In Proc. of Workshop on Uncertainty in Geometric Computations, 2001. Sheffield, UK.
[7] R. Farouki, C. Neff, and M. O'Connor. Automatic parsing of degenerate quadric-surface intersections. $A C M$ Transactions on Graphics, 8(3):174-203, 1989.

[8] P. Finsler. Über das Vorkommen definiter und semidefiniter Formen in Scharen quadratischer Formen Comment. Math. Helv., 9:188-192, 1936/1937.

[9] S. Fortune. Polyhedral modelling with exact arithmetic. In Proc. of ACM Symposium on Solid Modeling and Applications, pages 225-234, 1995.

[10] N. Geismann, M. Hemmer, and E. Schoemer. Computing the intersection of quadrics: exactly and actually. In Proc. of ACM Symposium on Computational Geometry, pages 264-273, 2001

[11] R. Goldman and J. Miller. Combining algebraic rigor with geometric robustness for the detection and calculation of conic sections in the intersection of two natural quadric surfaces. In Proc. of ACM Symposium on Solid Modeling Foundations and CAD/CAM Applications, pages 221-231, 1991.

[12] J. Harris. Algebraic Geometry: A First Course. Graduate Texts in Mathematics. Springer-Verlag, 1992.

[13] C. Hoffmann. Robustness in geometric computations. Journal of Computing and Information Science in Engineering, 1(2):143-156, 2001.

[14] J. Keyser, T. Culver, M. Foskey, and S. Krishnan. ESOLID - A system for exact boundary evaluation. In Proc. of 7th ACM Symposium on Solid Modeling and Applications, pp. $23-34,2002$

[15] J. Keyser, T. Culver, D. Manocha, and S. Krishnan. Efficient and exact manipulation of algebraic points and curves. Computer-Aided Design, 32(11):649-662, 2000. Special issue on robustness.

[16] J. Keyser, S. Krishnan, and D. Manocha. Efficient and accurate B-Rep generation of low degree sculptured solids using exact arithmetic: I - Representations, II Computation. Computer Aided Geometric Design, 16(9):841-859, 861-882, 1999.

[17] T. Lam. The Algebraic Theory of Quadratic Forms. W.A. Benjamin, Reading, MA, 1973

[18] J. Levin. A parametric algorithm for drawing pictures of solid objects composed of quadric surfaces. Communications of the ACM, 19(10):555-563, 1976.

[19] J. Levin. Mathematical models for determining the intersections of quadric surfaces. Computer Graphics and Image Processing, 11(1):73-87, 1979.

[20] J. Miller and R. Goldman. Geometric algorithms for detecting and calculating all conic sections in the intersection of any two natural quadric surfaces. Graphical Models and Image Processing, 57(1):55-66, 1995.

[21] MuPAD, the open computer algebra system, www.sciface.com

[22] F. Rouillier and P. Zimmermann. Efficient isolation of a polynomial real roots. Journal of Computational and Applied Mathematics, 2002. To appear. Technical Report RR-4113, Inria, 2001.

[23] C.-K. Shene and J. Johnstone. On the lower degree intersections of two natural quadrics. ACM Transactions on Graphics, 13(4):400-424, 1994.

[24] K. Sugihara. How to make geometric algorithms robust. IEICE Transactions on Information and Systems, E83-D(3):447-454, 2000.

[25] F. Uhlig. Simultaneous block diagonalization of two real symmetric matrices. Linear Algebra and Its Applications, $7: 281-289,1973$.

[26] F. Uhlig. A canonical form for a pair of real symmetric matrices that generate a nonsingular pencil. Linear Algebra and Its Applications, 14:189-209, 1976.

[27] I. Wilf and Y. Manor. Quadric-surface intersection curves: shape and structure. Computer-Aided Design, 25(10):633-643, 1993. 